\title{
Lymphocyte predominant exudative pleural effusions: a narrative review
}

\author{
Chang Li ${ }^{1}$, Farah I. Kazzaz ${ }^{2}$, Joanna M. Scoon ${ }^{2}$, Rosa M. Estrada-Y-Martin ${ }^{2}$, Sujith V. Cherian ${ }^{2}$ \\ ${ }^{1}$ Department of Internal Medicine, McGovern Medical School - UTHealth - The University of Texas Health Science Center at Houston, Houston, \\ TX, USA; ${ }^{2}$ Divisions of Critical Care, Pulmonary and Sleep Medicine, Department of Internal Medicine, McGovern Medical School - UTHealth - \\ The University of Texas Health Science Center at Houston, Houston, TX, USA \\ Contributions: (I) Conception and design: SV Cherian; (II) Administrative support: SV Cherian, RM Estrada-Y-Martin; (III) Provision of study \\ materials or patients: SV Cherian, RM Estrada-Y-Martin; (IV) Collection and assembly of data: All authors; (V) Data analysis and interpretation: All \\ authors; (VI) Manuscript writing: All authors; (VII) Final approval of manuscript: All authors. \\ Correspondence to: Sujith V. Cherian, MD, FCCP. University of Texas Health Science Center at Houston, 6431 Fannin street, Houston, TX 77030, \\ USA. Email: sujithvcherian@gmail.com; sujith.v.cherian@uth.tmc.edu.
}

Background and Objective: An estimated 1.5 million people develop pleural effusion each year in the United States. Although substantial progress has been made in understanding pleural effusion, establishment of a definite diagnosis usually necessitates thoracentesis with pleural fluid analysis. Lymphocyte predominant pleural effusion is defined as an exudative effusion with lymphocytes over $50 \%$ of the total white cell count. The purpose of this review article is to elaborate on the different causes of lymphocyte predominant pleural effusion and the unique biochemical and cellular characteristics of pleural fluid in these conditions.

Methods: This review is based on a PubMed search of English-language articles published between 1955 and 2020, using search terms including pleural effusion and lymphocyte predominant to generate a comprehensive literature list that highlights characteristics of pleural fluid in the different conditions associated with lymphocyte predominant pleural effusion.

Key Content and Findings: Tuberculosis and malignancy account for the majority of lymphocyte predominant pleural effusions. While less common, a number of conditions such as rheumatoid arthritis, post cardiac injury syndrome, chylothorax, sarcoidosis, yellow-nail syndrome, and uremia have also been described as potential causes of lymphocyte predominant pleural effusions.

Conclusion: Once an exudative effusion is identified, cell count and differential can further help narrow the differential diagnosis. Other diagnostic modalities including imaging and thoracoscopy with biopsy can also assist in achieving an accurate diagnosis. Management options include treatment for the underlying cause and/or symptoms, particularly in recurrent malignant pleural effusion.

Keywords: Pleural effusion; lymphocyte predominant; exudative effusion; malignant pleural effusion; tuberculous pleural effusion

Received: 23 May 2021; Accepted: 11 November 2021; Published: 30 January 2022.

doi: $10.21037 /$ shc-21-11

View this article at: https://dx.doi.org/10.21037/shc-21-11

\section{Introduction}

Pleural effusion is a common presentation of many pulmonary and systemic diseases, particularly those with high mortality such as malignancy and infections. It is estimated that 1.5 million people develop pleural effusions each year in the United States (1). While various diagnostic and treatment modalities have been developed in recent years, the analysis of pleural fluid remains the mainstay of the diagnostic approach to pleural effusions.

The initial step in the evaluation of a pleural effusion is to determine whether it is a transudate or an exudate. Light's 
criteria still remains the principal method to make this differentiation by utilizing protein and lactate dehydrogenase levels both in the pleural fluid and blood (2). Light's criteria is defined by the following: (I) Pleural fluid protein: serum protein $>0.5$, (II) Pleural fluid lactate dehydrogenase (LDH): serum $\mathrm{LDH}>0.6$ and (III) pleural fluid LDH greater than $2 / 3$ of the upper limit of serum LDH. To define exudative effusion, the effusion should meet at least one of these criteria (3). Transudates are caused by an imbalance between hydrostatic and oncotic pressures, as found commonly in heart failure and cirrhosis. Exudates are caused by inflammation leading to increased capillary leakage, impaired lymphatic drainage due to obstruction, or both (4). However, Light's criteria can misclassify nearly $25 \%$ of transudates, particularly in patients with heart failure treated with diuretics. Such misclassification requires additional testing with fluid-serum albumin gradient and natriuretic peptides to confirm the diagnosis. Thus, this highlights that other components of the fluid analysis, including routine biochemical and cytological examination, are crucial and can further increase diagnostic accuracy. Noppen et al. analyzed the cellular contents of the pleural fluid in normal individuals and demonstrated that the mean white cell counts (WBC) was 1,716 cells $/ \mathrm{mm}^{3}$, of which approximately $75 \%$ of the cells are macrophages. Lymphocytes account for roughly $20 \%$ of the cell count. Mesothelial cells, neutrophils, and eosinophils together account for the remaining 5\% (5). Cell counts and differential are helpful in evaluation of the underlying cause of a pleural effusion and are routinely performed. The predominance of a WBC population reflects the mechanism of pleural injury, as well as the duration of an effusion. Light and colleagues (6) demonstrated that certain conditions were closely associated with the predominance of a particular cell line. A lymphocyte predominant exudative effusion, defined as lymphocytes more than $50 \%$ of the total WBC, is often suggestive of a chronic pleural process. In contrast, neutrophilic predominance is more frequently observed in an acute response. Other cells lines can provide additional clues. Conditions associated with a very high red blood cell $\left(>100,000 \mathrm{cell} / \mathrm{mm}^{3}\right)$ count include malignancy, pulmonary infarction, and trauma. Elevated eosinophil counts ( $>10 \%$ of the WBC count) are most frequently observed in pneumothorax and hemothorax (7) (Figure 1).

In this review, we aim to summarize a comprehensive list of conditions associated with lymphocyte-predominant exudates and discuss the management in each of these conditions. We present the following article in accordance with the Narrative Review reporting checklist (available at https://shc.amegroups.com/article/view/10.21037/shc-21$11 / \mathrm{rc})$.

\section{Methods}

We conducted a search of PubMed for publications with the search terms of pleural effusion and lymphocyte predominant (Table 1). We searched for relevant English-language articles published between January 1, 1955, and December 31, 2020 with a focus on systematic reviews, narrative reviews, and clinical practice guidelines to compile a comprehensive list. Additional publications were identified through bibliography. Relevant studies were reviewed by the authors and their results were summarized in this article.

\section{Tuberculous pleurisy}

Tuberculosis (TB) is a worldwide health issue affecting approximately 10 million individuals each year (8). The incidence of tuberculous pleural effusion (TPE) is seen to vary depending on its regional prevalence, with as many as $20-25 \%$ of patients with TB presenting with pleural effusion in Spain while in the US, the prevalence is 3-4\% of patients with TB (9). The pathogenesis of TPE was initially thought to be a delayed hypersensitivity reaction to Mycobacterial antigens in the pleural space. This theory was further supported by the low yield from solid culture and the presence of lymphocytes in TPF. However, with the improved yield of liquid culture media, Mycobacterium tuberculosis can be isolated and TPE is thought to occur as a result of a paucibacillary infection in the pleural space spread from pulmonary lesions (10). The effusions are usually unilateral, small to moderate size, and self-limited (11). However, if left untreated, development of active TB (open pulmonary TB) is seen in $43-65 \%$ of patients within the subsequent years $(12,13)$. Pulmonary lesions are frequently observed with TPE due to disseminated TB to the pleura. Computed tomography (CT) is more sensitive than chest radiography to detect associated parenchymal lesions in $80 \%$ of TPE $(10,14,15)$.

On TPE pleural fluid analysis, routine chemistry often shows $\mathrm{pH}$ less than 7.4, glucose levels greater than $60 \mathrm{mg} / \mathrm{dL}$ similar to serum, and elevated protein levels above $4.0 \mathrm{~g} / \mathrm{dL}$. High lymphocyte count, commonly exceeding $80 \%$ of the WBC is usually seen. During the acute phase, TPE may present with neutrophil predominant effusions. Recent studies have shown that approximately $90 \%$ of TPE 


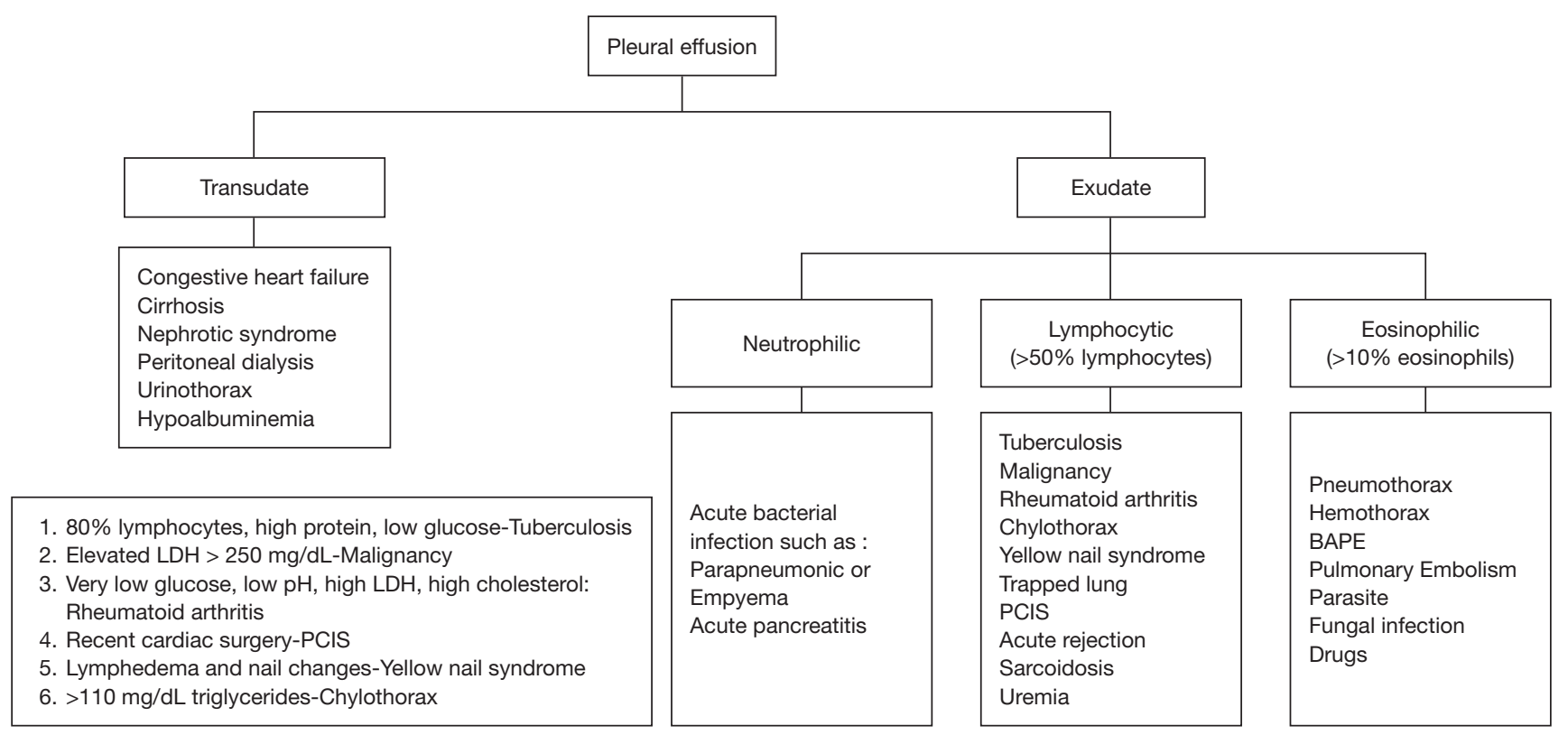

Figure 1 Algorithm showing classification of pleural effusions and further differentiation of exudative effusion based on cell type. PCISpost-cardiotomy injury syndrome; ESRD, end-stage renal disease; BAPE, benign asbestos pleural effusion.

Table 1 The search strategy summary

\begin{tabular}{ll}
\hline Items & Specification \\
\hline Date of Search (specified to date, month and year) & Nov 18,2020 \\
Databases and other sources searched & PubMed \\
$\begin{array}{l}\text { Search terms used (including MeSH and free text search terms and filters) } \\
\text { Note: please use an independent supplement table to present detailed search } \\
\text { strategy of one database as an example }\end{array}$ & Search strategy listed as below \\
Timeframe & \\
Inclusion and exclusion criteria (study type, language restrictions etc.) & Jan 1, 1955 to Dec 31, 2020 \\
$\begin{array}{l}\text { Selection process (who conducted the selection, whether it was conducted } \\
\text { independently, how consensus was obtained, etc.) }\end{array}$ & Inclusion criteria listed as below \\
Any additional considerations, if applicable & All authors reviewed and selected studies relevant \\
to lymphocyte predominant pleural effusion
\end{tabular}

\footnotetext{
"Key words include "lymphocytes" and "pleural effusion." Mesh terms were used for initial search. Example: ("Lymphocytes"[MeSH Terms:noexp] OR "Lymphocyte Count”[MeSH Terms] OR ("Iymphocyt*”[Title/Abstract] OR "lymphocyt"”[Text Word])) AND (“Pleural Effusion"[MeSH Terms] OR ("pleural effusion*"[Title/Abstract] OR "pleural effusion*”[Text Word])). **Inclusion criteria were applied to English literature using the search strategy shown in this table. The articles consist of systematic and narrative reviews with clinical data (cellular and biochemical characteristics of pleural effusion, etc.)
}

are lymphocyte predominant and $10 \%$ are neutrophil predominant (16,17). HIV status does not change the cell distribution in TPE (18). In fact, pleural effusions are more common in patients with HIV and concomitant pulmonary TB. TPE rarely contains $>5 \%$ mesothelial cells, due to the intense pleural inflammation which prevents mesothelial shedding from the pleural membranes. Furthermore, adenosine deaminase (ADA) levels can be useful in identifying TPE from other causes with a cut-off value above $40 \mathrm{U} / \mathrm{L}$. False positive and false negative results do occur with the test, as its positive and negative predictive values change in relation to the prevalence of TB. In 

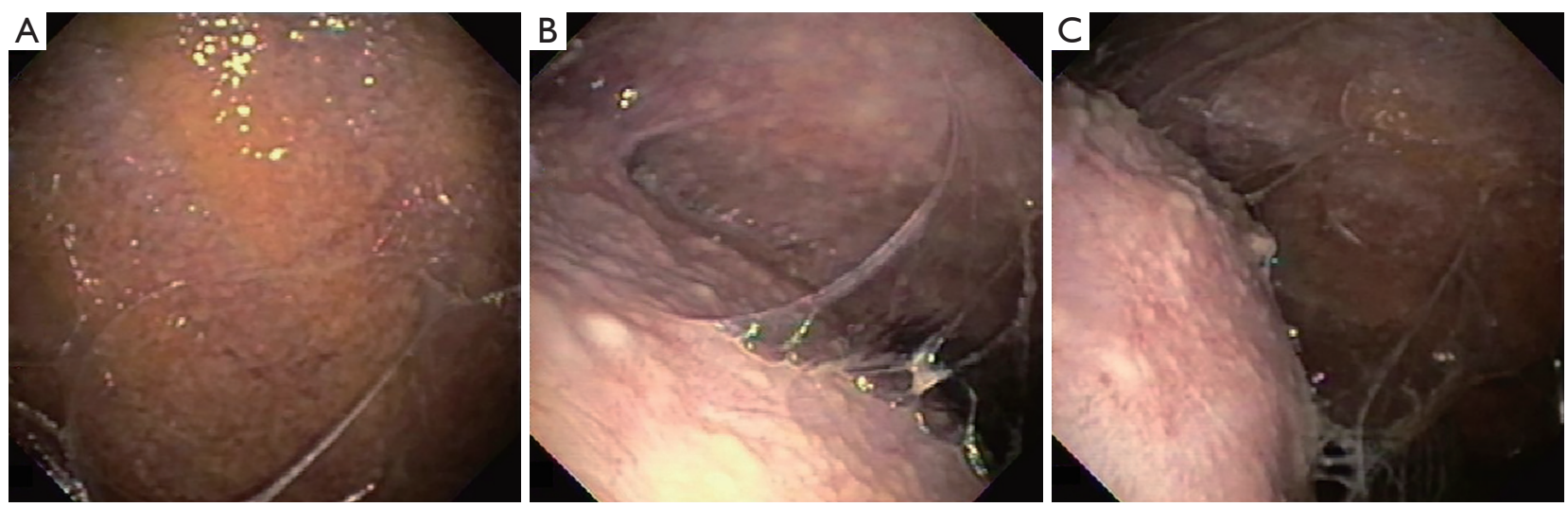

Figure 2 Medical thoracoscopy images on a patient presenting with unilateral lymphocyte predominant pleural effusion eventually diagnosed as Tuberculous pleural effusion. (A) Images on medical thoracoscopy in a patient with tuberculous pleural effusion. Note the diffuse granular appearance of nodules distributed over the parietal pleura. (B) Medical thoracoscopy images in same patient showing adhesions and discrete yellowish-white nodules, commonly referred to as sago-like nodules distributed over the parietal pleura and the diaphragm. (C) Medical thoracoscopy in same patients—where more adhesions are seen along with diffuse sago-like nodules.

endemic areas, a combination of an ADA level over $40 \mathrm{U} / \mathrm{L}$ and a lymphocyte-to-neutrophil ratio greater than 0.75 is sufficient for a presumptive diagnosis of TPE (19). In non-endemic areas, although the positive predictive value decreases, ADA levels less than $40 \mathrm{U} / \mathrm{L}$ has a high negative predictive value for TPE (20). Other tests that may be useful include pleural fluid interferon-gamma concentration and lysozyme concentration, which are of similar diagnostic utility; however not frequently utilized likely due to the low cost of ADA. Additionally, within the US, the FDA has not validated interferon gamma and lysozyme concentrations in pleural effusions $(1,15)$.

Besides pleural biopsy, other tests are generally not helpful when fluid analysis is inconclusive. Sputum culture has a low diagnostic yield ranging between 0 and $30 \%$. Induced sputum culture has been shown to have a higher yield of $52 \%$ even in patients with normal radiograph. Nucleic acid amplification of induced sputum culture in patients with TPE only provides approximately $25 \%$ of yield. Smear and culture of the pleural fluid yield less than $10 \%$ and $30 \%$ in TPE, respectively, although positive HIV status and neutrophil predominance may increase the yield $(9,16)$. Pleural biopsy may be obtained through various modalities including ultrasound-guided biopsy or thoracoscopy. The presence of caseating granulomas and acid-fast bacilli are diagnostic of TPE. Tissue biopsy should also be sent for culture to identify organism and drug susceptibility. Closed pleural biopsy with pathological and microbiological evaluation has been reported to have an yield of up to $91 \%$ for TB, while biopsies through thoracoscopy (Figure 2) has a diagnostic yield of up to $100 \%$ in TPE (21).

Treatment of TPE involves a 6-month course of antituberculosis drugs including an intensive phase of rifampin, isoniazid, pyrazinamide and ethambutol (HRZE) for two months, followed by 4 months of rifampin and isoniazid, similar to treatment of pulmonary tuberculosis (15). Role of corticosteroids in TPE is controversial (1).

\section{Malignant pleural effusion}

Malignancy is the second leading cause of exudative effusions, preceded by parapneumonic effusions. Malignant pleural effusion (MPE) typically presents as a large unilateral effusion, but certain conditions, such as pleural mesothelioma, can present small unilateral effusion without significant symptoms. The presence of a pleural effusion in the setting of malignancy typically indicates a poor prognosis. Median survival ranges from 3-12 months depending on the type of malignancy and stage at diagnosis (22). Cancers of the lung, breast and lymphoma account for almost $75 \%$ of MPE. The diagnosis of MPE remains crucial as it signifies metastases and upstages the malignancy.

The initial diagnostic test for suspected MPE is thoracentesis with fluid analysis and cytology. Routine chemistry commonly shows low pH less than 7.3 , low glucose levels less than $60 \mathrm{mg} / \mathrm{dL}$ in $20-30 \%$ of patients $(23,24)$ and are almost always exudates based on LDH criteria. A transudate may be seen in around $5 \%$ of 
Table 2 Types of tumor associated with MPE

\begin{tabular}{ll}
\hline Men & Women \\
\hline Lung & Breast \\
Lymphoma/leukemia & Ovarian/mullerian origin \\
Genitourinary & Lung \\
Gastrointestinal tract & Lymphoma/leukemia \\
Melanoma & Gastrointestinal \\
Mesothelioma & Melanoma \\
\hline
\end{tabular}

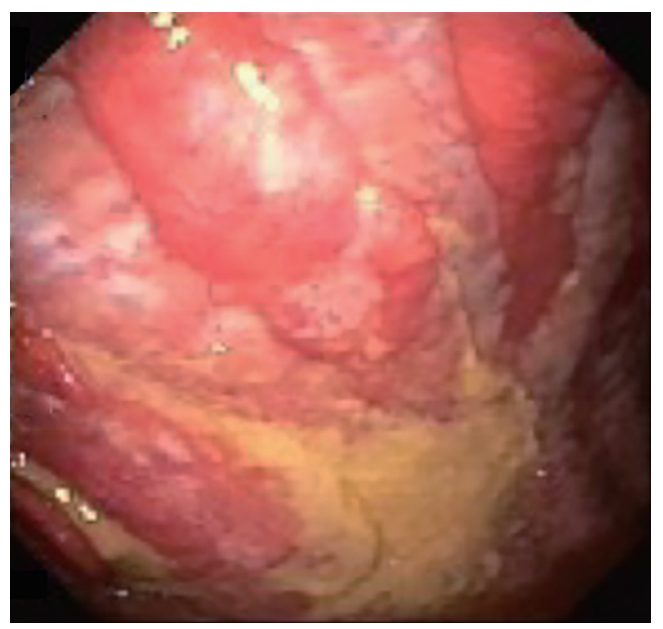

Figure 3 Images on medical thoracoscopy in a patient with malignant pleural effusion due to metastatic breast cancer. Notice the thickened parietal pleura and nodules distributed.

cases (25). Diagnostic yield of cytology is approximately $60 \%$ on an initial thoracentesis sample (22). A repeat thoracentesis may provide additional yield of up to $27 \%$ (26), although the data have been inconsistent in this regard. The low yield is partially due to the paucity of metastatic cells in the pleural space and overcrowding of inflammatory cells. Moreover, beyond a second attempt at thoracentesisfurther aspirations are unlikely to be diagnostic (26). The distinction between MPE and paramalignant effusions is also important. Paramalignant effusion results from local or systemic effects of a tumor without direct involvement of the pleura. Common examples include compression of the bronchi or the lymphatic system. Cytology is negative because infiltration to the pleura has not occurred (27). Furthermore, the diagnostic yield of cytology varies with the type of tumor involved. Adenocarcinoma is most frequently detected on cytology, whereas squamous cell carcinoma and mesothelioma are less frequently observed $(28,29)$. Cytological diagnosis of lymphoma is possible in $25 \%$ of patients with Hodgkin's lymphoma and $50-60 \%$ of patients with non-Hodgkin's Lymphoma (1). Gender may also play a role in the distribution of tumor types associated with MPE, as noted lung and lymphoma/leukemia more common in men and breast and gynecologic tumors more common in women (Table 2).

Given the low sensitivity of cytological evaluation of pleural fluid for malignancy, other diagnostic modalities should be pursued. Tumor markers have been shown to have limited utility as initial evaluation due to the high cutoff values for specificity (30). Pleural biopsy can be obtained via different modalities, including CT-guided or ultrasound-guided needle biopsy. In recent years, biopsy obtained via thoracoscopy, particularly in the form of pleuroscopy (Figure 3), has been shown to have a sensitivity more than $90 \%$, similar to that of videoassisted thoracoscopy (31). While treating the underlying malignancy with appropriate chemotherapy may lead to the resolution of MPE (especially in the setting of small cell lung cancer and lymphoma), recurrence of MPE is fairly common. A low glucose and $\mathrm{pH}$ predict a shorter survival, as they are usually associated with increased tumor burden within the pleural space (24). For persistent and symptomatic MPE repeat therapeutic thoracentesis may be appropriate in patients with an anticipated short life expectancy. However, in most cases either an indwelling pleural catheter or chemical pleurodesis would be necessary due to the recurrent nature of these effusions. Choice of either therapy is largely governed by patient preference and available local expertise. In patients with entrapped or trapped lung and symptomatic MPE, indwelling pleural catheters present the only available option $(27,32)$. Fluid aspiration is not recommended in small and asymptomatic pleural effusions. Moreover, if thoracentesis is not associated with symptom improvement, further attempts at fluid drainage should not be performed (1).

\section{Rheumatoid pleurisy}

Rheumatoid arthritis (RA) is a systemic disease that affects both the joints and extra-articular sites, particularly the pleura as the most common extra-articular manifestation in the chest (7). RA-associated pleural effusion is more frequently seen in men and those with rheumatoid nodules and high titers of rheumatoid factor. Most of the pleural effusions are small and unilateral, and only 
$3-5 \%$ of the patients develop large pleural effusions with symptoms including pleuritic chest pain and dyspnea (33). Pleuropulmonary complications, such as empyema and pneumothorax, may occur when subpleural rheumatoid nodules become necrotic and rupture, leading to the formation of bronchopleural fistula. Furthermore, treatment for RA tends to put these patients at higher risk for infection due to immunosuppression. Certain medications, such as methotrexate and sulfasalazine, have been linked with numerous pleuropulmonary presentations as well $(33,34)$. A thoracentesis is thus necessary in a symptomatic patient due to the multiple risk factors associated with RA.

The characteristics of RA-associated pleural effusion vary according to the duration of the pleural fluid. Cell count is generally above $1,000 \mathrm{cell} / \mathrm{mm}^{3}$ consistent with active inflammation. Neutrophils predominate in early effusions, which is then replaced by lymphocytes in 1 to 2 weeks (35). Elevated eosinophil counts rarely occur. Mesothelial cells are typically not seen in the pleural fluid. Biochemical parameters of the pleural fluid show low $\mathrm{pH}$, very low glucose levels (a characteristic feature with pleural to serum glucose ratio less than 0.5 (36), elevated LDH levels, and high cholesterol levels (more than $65 \mathrm{mg} / \mathrm{dL}$ ) (37). Acidosis and low glucose level reflect the active inflammatory process with the accumulation of lactic acids and consumption of glucose. High cholesterol concentration and cholesterol crystals can also be found in RA- associated pleural effusions due to pleural thickening from chronic inflammation. High cholesterol level can cause a milky appearance, resulting in a "pseudochylous" pleural fluid. Among the causes of pseudochylothorax, TB and RA are the most frequent ones.

The diagnostic approach to RA-associated pleural effusion should be prioritized to rule out infection. The possibility of empyema and TPE is high in a symptomatic patient given their risk factors. Cytology provides additional information with characteristic findings, such as elongated spindle cells, in the pleural effusion $(33,38)$. Pleural biopsy is generally not needed unless there is suspicion for malignancy or TB. Biopsy findings include the presence of rheumatoid nodules and the replacement of mesothelial cells by epitheloid cells. Little information is available on the efficacy of therapy in rheumatoid pleural disease. Corticosteroids have been used with varying success and the role of intrapleural corticosteroids is also uncertain $(39,40)$. The natural course of rheumatoid pleural effusions is variable-with the majority of effusions showing spontaneous resolution. However, a small percentage of patients may develop pleural fibrosis for which decortication should be considered in dyspneic patients (33).

\section{Coronary artery bypass graft (CABG)}

It is estimated that over 600,000 patients undergo CABG annually each year in the United States (41). Pleural effusions after CABG and other cardiothoracic surgeries are common, with an incidence ranging between $42 \%$ to $89 \%$ (42). The wide range of incidence is likely due to the different techniques employed by the studies, such as different imaging modalities for diagnosis. Most of the pleural effusions are small, left-sided, and not clinically significant. However, less than $10 \%$ of patients may develop a large effusion (more than $25 \%$ of the hemithorax) with symptoms, such as dyspnea $(43,44)$ and is more commonly associated with internal mammary grafts as compared to saphenous vein grafts (41). Labadi and colleagues demonstrated that these patients more frequently developed postoperative complications which resulted in longer hospital and ICU stay. The same cohort was also noted to have more severe comorbidities and require more complex surgeries, including combined procedure with valve replacement and prolonged surgery. Most large pleural effusions following CABG occur in the early postoperative period and often require thoracentesis to manage symptoms.

Pleural effusions after CABG are categorized as early (within the first 30 days of the procedure) and late (after first 30 days of the procedure) based on the features of pleural fluid analysis (42). In several studies, early and late effusions were found to be exudates. Characteristic features of early effusions consisted of elevated erythrocyte count, high $\mathrm{LDH}$ levels, and a predominance in neutrophils (42-44). Late effusions were shown to have less erythrocyte count and a predominance of lymphocytes. Given these findings, the mechanism for the pleural effusions is likely secondary to be trauma-related and immune-mediated in early and late effusions, respectively. Additionally, reports have shown other possible mechanisms, such as heart failure or injuries to thoracic duct and its tributaries, leading to the formation of pleural effusions after CABG $(7,45,46)$. Since the focus of this article is on lymphocyte predominant pleural effusions, late pleural effusions will be discussed within this section. Post cardiac injury syndrome (PCIS) refers to the development of chest pain, fever, pulmonary opacities, left sided pleural effusions after myocardial infarction, cardiac surgery, penetrating chest trauma and pacemaker placement (47). Although it may be seen as early as 3 days after the antecedent event, it usually develops at an average 


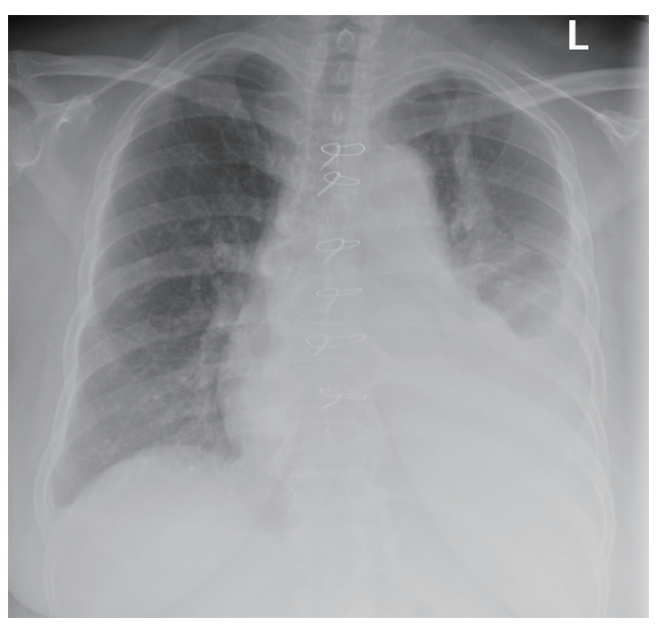

Figure 4 Chest $\mathrm{X}$-ray in a patient who presented with complaints of shortness of breath 3 weeks after surgery for repair of aortic dissection. Pleural fluid studies showed a lymphocyte predominant (52\%) exudative pleural effusion consistent with PCIS.

of 3 weeks post procedure (Figure 4). PCIS is relatively common and seen in almost $30 \%$ of patients post cardiac surgery. Late pleural effusion after CABG and PCIS share similar pathophysiology, which is believed to be secondary to an exaggerated immune response to the inflammatory changes of the pleura and myocardial antigens released after myocardial injury. Overall the clinical manifestation of late pleural effusion following cardiac surgery overlaps with that of PCIS, except that fever and chest pain are perhaps less common in late pleural effusion.

PCIS related pleural effusions are usually clear, yellow exudative effusions, which are lymphocyte predominant later in the course, typically within 10 days after onset of symptoms $(42,45,47)$. It is a diagnosis of exclusion and other causes such as parapneumonic effusions, congestive heart failure and pulmonary embolism should be excluded (1). Antimyocardial antibodies are a sensitive and specific confirmatory test (48). Most patients have resolution of PCIS without clinically significant sequelae- although it may contribute to the development of entrapped and trapped lung eventually in rare circumstances (45). Thus close follow up is essential in these patients. PCIS usually responds to treatment with anti-inflammatory agents such as NSAIDs or aspirin. Colchicine has been shown to reduce incidence of pleural effusions when given after cardiac surgery (49). In refractory cases, a course of glucocorticoids may be necessary (50) but their withdrawal often results in recurrence. Moreover, given the deleterious effects on wound healing- it should only be used as last resort (45).

\section{Chylothorax}

Chylothorax is a pleural effusion that results from leakage of chyle into the pleural space when the thoracic duct or its tributaries are disrupted. Therefore, understanding the anatomy of the lymphatic drainage system can help determine the underlying mechanism and location that cause chylothorax. Chylothorax is potentially life-threatening with a mortality up to $50 \%$ when treated conservatively (51). The underlying etiologies can be classified as traumatic and non-traumatic (52). Traumatic causes include not only chest trauma, but also iatrogenic causes such as thoracic surgeries (e.g., esophagectomy, heart and lung transplant), central line placement, and pacemaker implantation. Nontraumatic causes include both malignant and non-malignant disorders. Malignancy, particularly lymphoma, is the most common cause of non-traumatic chylothorax. Several nonmalignant causes have been described and some of these include sarcoidosis, retrosternal goiter, superior vena cava thrombosis, congenital duct abnormalities, and lymphatic disorders including yellow nail syndrome and low grade malignancies such as lymphangioleiomyomatosis (53).

Chylothorax should be suspected if the pleural fluid appears milky or cloudy on gross examination of the pleural fluid. However, non-milky appearance including serous, bloody, or serosanguinous is also common so the clinical context and surgical history is crucial in helping ascertain a diagnosis of chylothorax (54). Analysis of pleural fluid attained from a thoracentesis or chest tube is essential and should include lipid testing. A chylothorax is expected to have an elevated level of triglycerides, usually greater than $110 \mathrm{mg} / \mathrm{dL}$, and a cholesterol level less than $200 \mathrm{mg} / \mathrm{dL}$. Empyema and pseudochylothorax can have similar milky appearance to chylothorax, therefore further testing with centrifugation of the sample and cholesterol level are helpful to make the differentiation. Studies have shown that the majority of cases of chylothorax are exudates with lymphocyte predominance $(54,55)$. However, transudates do occur, particularly when there is another co-existing condition such as heart failure or cirrhosis. Routine chemistry typically consists of normal to high $\mathrm{pH}$, normal glucose $(>60 \mathrm{mg} / \mathrm{dL})$, elevated protein level, and low $\mathrm{LDH}$. Protein level is often above $3.0 \mathrm{~g} / \mathrm{dL}$ possibly due to reabsorption of water and small solutes from the chylous effusion. In contrast, LDH level remains in the transudative range because of its larger molecular weight as a barrier to 
extravasate from the capillaries.

Management of chylothorax should be directed at definitive treatment of underlying etiology to prevent further formation (56). Prolonged chest tube placement in chylothorax can lead to complications associated with immunosuppression and profound malnutrition due to loss of protein, fat, electrolytes, and immunoglobulins.

\section{Sarcoidosis}

Sarcoidosis is a systemic disease characterized by noncaseating granulomas in affected organs. While pulmonary involvement is the most common manifestation of sarcoidosis, pleural involvement is considered rare. When sarcoidosis affects the pleura, a wide range of presentations including pleural effusion, pneumothorax, pleural thickening, or chylothorax can be seen. Huggins and colleagues demonstrated $2.8 \%$ of prevalence of pleural effusion in patients with sarcoidosis. However, only $1.1 \%$ of those cases ( 2 out of 181) were directly caused by pleural sarcoidosis (57). Therefore, other etiologies should be sought in those patients who have a known history of sarcoidosis and a clinical presentation of pleural effusion.

Pleural effusion in sarcoidosis has been reported as both exudative and transudative. This is partially due to the normal pleural LDH concentrations and elevated pleural protein levels, in the range of $4.0 \mathrm{~g} / \mathrm{dL}(57,58)$. Additionally, the cell count tends to be low with a predominance of lymphocytes with a high proportion over $80 \%$. Chylothorax has been reported rarely in sarcoidosis (58). Corticosteroid therapy in pleural sarcoidosis is associated with rapid amelioration of symptoms and resolution of pleural effusion (59).

\section{Yellow Nail Syndrome}

Yellow nail syndrome is a rare disorder characterized by pleural effusions, lymphedema and unusual appearing nails that are characteristically yellow, slow growing with absence of cuticle. The nail may have other abnormalities such as onycholysis, increased curvature, or lack lunulae. Impaired lymphatic system is thought to play a role in the development of this disorder and it remains unclear whether the disorder is sporadic or inherited. Other respiratory conditions have been associated with yellow nail syndrome include bronchiectasis, recurrent pneumonia, and chronic sinusitis. Pleural fluid analysis is typically consistent with a lymphocytic predominant exudate (60). Yellow nails syndrome has also been associated with chylothorax in up to $30 \%$ of these patients with pleural effusions (61). No specific therapy exists for this entity and in patients with a large symptomatic pleural effusion, pleurodesis either by chemical or mechanical means should be considered $(1,61)$.

\section{Uremia}

Patients with chronic kidney disease and end-stage renal disease can develop pleural effusions from indirect causes such as infection, volume overload, malignancy, or heart failure. However, they can also develop pleuritis, akin to the development of pericarditis with resultant development of pleural effusions as a direct effect of uremia. This may be seen in around $3 \%$ of patients with uremia and are frequently bilateral. Pleural fluid analysis is expected to be consistent with a lymphocytic predominant pleural exudate which is commonly serosanguineous or frankly hemorrhagic. Symptoms may include cough, fever, dyspnea and/or chest pain $(62,63)$. Uremic pleural effusion is a diagnosis of exclusion and it is important to evaluate for other causes of pleural effusions as described above.

Pleural biopsy reveals chronic fibrinous pleuritis. The treatment of choice for uremic pleural effusions is renal replacement therapy with hemodialysis which causes resolution of the pleural effusion in approximately $75 \%$ of cases in 4 to 6 weeks; while in the remaining $25 \%$ of casesthe effusion progresses, persists or recurs. Complications of uremic pleuritis if left untreated include the development of progressive pleural thickening with resultant severe ventilatory impairment, which may require decortication $(64,65)$.

\section{Conclusion}

Encountering a pleural effusion in clinical practice should start with a comprehensive evaluation of the patient including a detailed history and physical examination, along with evaluation of radiological images. Thoracentesis with pleural fluid analysis is often required to reach a definitive diagnosis. While distinction on analysis between exudate and transudate remains the first step on analysis of pleural fluid, cell count and differential help narrow the differential diagnoses significantly. Lymphocyte predominant exudative pleural effusions have a narrow list of differentials with its own unique management approaches and knowledge of these entities will be of benefit in routine clinical practice. 


\section{Acknowledgments}

Funding: None.

\section{Footnote}

Reporting Checklist: The authors have completed the Narrative Review reporting checklist. Available at https:// shc.amegroups.com/article/view/10.21037/shc-21-11/rc

Peer Review File: Available at https://shc.amegroups.com/ article/view/10.21037/shc-21-11/prf

Conflicts of Interest: All authors have completed the ICMJE uniform disclosure form (available at https://shc.amegroups. com/article/view/10.21037/shc-21-11/coif). SVC serves as an unpaid editorial board member of Shanghai Chest from Jun 2020 to May 2022. The other authors have no conflicts of interest to declare.

Ethical Statement: The authors are accountable for all aspects of the work in ensuring that questions related to the accuracy or integrity of any part of the work are appropriately investigated and resolved.

Open Access Statement: This is an Open Access article distributed in accordance with the Creative Commons Attribution-NonCommercial-NoDerivs 4.0 International License (CC BY-NC-ND 4.0), which permits the noncommercial replication and distribution of the article with the strict proviso that no changes or edits are made and the original work is properly cited (including links to both the formal publication through the relevant DOI and the license). See: https://creativecommons.org/licenses/by-nc-nd/4.0/.

\section{References}

1. Light RW. Pleural diseases. 6th ed. Philadelphia, PA Lipincott Williams \& Wilkins; 2013.

2. Feller-Kopman D, Light R. Pleural Disease. N Engl J Med 2018;378:740-51.

3. Light RW, Macgregor MI, Luchsinger PC, et al. Pleural effusions: the diagnostic separation of transudates and exudates. Ann Intern Med 1972;77:507-13.

4. Sahn SA. Getting the most from pleural fluid analysis. Respirology 2012;17:270-7.

5. Noppen M, De Waele M, Li R, et al. Volume and cellular content of normal pleural fluid in humans examined by pleural lavage. Am J Respir Crit Care Med 2000;162:1023-6.

6. Light RW, Erozan YS, Ball WC Jr. Cells in pleural fluid. Their value in differential diagnosis. Arch Intern Med 1973;132:854-60.

7. Sahn SA, Huggins JT, San Jose E, et al. The Art of Pleural Fluid Analysis. Clinical Pulmonary Medicine 2013;20:77-96.

8. WHO. Global tuberculosis report. 2019. Available online: https://apps.who.int/iris/handle/10665/329368.

9. Gopi A, Madhavan SM, Sharma SK, et al. Diagnosis and treatment of tuberculous pleural effusion in 2006. Chest 2007;131:880-9.

10. Vorster MJ, Allwood BW, Diacon AH, et al. Tuberculous pleural effusions: advances and controversies. J Thorac Dis 2015;7:981-91.

11. Jolobe OM. Atypical tuberculous pleural effusions. Eur J Intern Med 2011;22:456-9.

12. PATIALA J. Initial tuberculous pleuritis in the Finnish armed forces in 1939-1945 with special reference to eventual postpleuritic tuberculosis. Acta Tuberc Scand Suppl 1954;36:1-57.

13. ROPER WH, WARING JJ. Primary serofibrinous pleural effusion in military personnel. Am Rev Tuberc 1955;71:616-34.

14. Kim HJ, Lee HJ, Kwon SY, et al. The prevalence of pulmonary parenchymal tuberculosis in patients with tuberculous pleuritis. Chest 2006;129:1253-8.

15. Light RW. Update on tuberculous pleural effusion. Respirology 2010;15:451-8.

16. Bielsa S, Palma R, Pardina M, et al. Comparison of polymorphonuclear- and lymphocyte-rich tuberculous pleural effusions. Int J Tuberc Lung Dis 2013;17:85-9.

17. Choi H, Chon HR, Kim K, et al. Clinical and Laboratory Differences between Lymphocyte- and NeutrophilPredominant Pleural Tuberculosis. PLoS One 2016;11:e0165428.

18. Relkin F, Aranda CP, Garay SM, et al. Pleural tuberculosis and HIV infection. Chest 1994;105:1338-41.

19. Burgess LJ, Maritz FJ, Le Roux I, et al. Combined use of pleural adenosine deaminase with lymphocyte/neutrophil ratio. Increased specificity for the diagnosis of tuberculous pleuritis. Chest 1996;109:414-9.

20. Lee YC, Rogers JT, Rodriguez RM, et al. Adenosine deaminase levels in nontuberculous lymphocytic pleural effusions. Chest 2001;120:356-61.

21. Diacon AH, Van de Wal BW, Wyser C, et al. Diagnostic tools in tuberculous pleurisy: a direct comparative study. Eur Respir J 2003;22:589-91. 
22. Psallidas I, Kalomenidis I, Porcel JM, et al. Malignant pleural effusion: from bench to bedside. Eur Respir Rev 2016;25:189-98.

23. Martínez-Moragón E, Aparicio J, Sanchis J, et al. Malignant pleural effusion: prognostic factors for survival and response to chemical pleurodesis in a series of 120 cases. Respiration 1998;65:108-13.

24. Rodríguez-Panadero F, López Mejías J. Low glucose and $\mathrm{pH}$ levels in malignant pleural effusions. Diagnostic significance and prognostic value in respect to pleurodesis. Am Rev Respir Dis 1989;139:663-7.

25. Ashchi M, Golish J, Eng P, et al. Transudative malignant pleural effusions: prevalence and mechanisms. South Med J 1998;91:23-6.

26. Garcia LW, Ducatman BS, Wang HH. The value of multiple fluid specimens in the cytological diagnosis of malignancy. Mod Pathol 1994;7:665-8.

27. Asciak R, Rahman NM. Malignant Pleural Effusion: From Diagnostics to Therapeutics. Clin Chest Med 2018;39:181-93.

28. Dermawan JKT, Policarpio-Nicolas ML. Malignancies in Pleural, Peritoneal, and Pericardial Effusions. Arch Pathol Lab Med 2020;144:1086-91.

29. Johnston WW. The malignant pleural effusion. A review of cytopathologic diagnoses of 584 specimens from 472 consecutive patients. Cancer 1985;56:905-9.

30. Porcel JM, Vives M, Esquerda A, et al. Use of a panel of tumor markers (carcinoembryonic antigen, cancer antigen 125, carbohydrate antigen 15-3, and cytokeratin 19 fragments) in pleural fluid for the differential diagnosis of benign and malignant effusions. Chest 2004;126:1757-63.

31. Rahman NM, Ali NJ, Brown G, et al. Local anaesthetic thoracoscopy: British Thoracic Society Pleural Disease Guideline 2010. Thorax 2010;65 Suppl 2:ii54-60.

32. Ferreiro L, Suárez-Antelo J, Valdés L. Pleural procedures in the management of malignant effusions. Ann Thorac Med 2017;12:3-10.

33. Balbir-Gurman A, Yigla M, Nahir AM, et al. Rheumatoid pleural effusion. Semin Arthritis Rheum 2006;35:368-78.

34. Bintcliffe OJ, Lee GY, Rahman NM, et al. The management of benign non-infective pleural effusions. Eur Respir Rev 2016;25:303-16.

35. Avnon LS, Abu-Shakra M, Flusser D, et al. Pleural effusion associated with rheumatoid arthritis: what cell predominance to anticipate? Rheumatol Int 2007;27:919-25.

36. Lillington GA, Carr DT, Mayne JG. Rheumatoid pleurisy with effusion. Arch Intern Med 1971;128:764-8.

37. Shaw M, Collins BF, Ho LA, et al. Rheumatoid arthritis- associated lung disease. Eur Respir Rev 2015;24:1-16.

38. Komarla A, Yu GH, Shahane A. Pleural effusion, pneumothorax, and lung entrapment in rheumatoid arthritis. J Clin Rheumatol 2015;21:211-5.

39. Russell ML, Gladman DD, Mintz S. Rheumatoid pleural effusion: lack of response to intrapleural corticosteroid. J Rheumatol 1986;13:412-5.

40. Chapman PT, O'Donnell JL, Moller PW. Rheumatoid pleural effusion: response to intrapleural corticosteroid. J Rheumatol 1992;19:478-80.

41. Light RW. Pleural effusions after coronary artery bypass graft surgery. Curr Opin Pulm Med 2002;8:308-11.

42. Sadikot RT, Rogers JT, Cheng DS, et al. Pleural fluid characteristics of patients with symptomatic pleural effusion after coronary artery bypass graft surgery. Arch Intern Med 2000;160:2665-8.

43. Light RW, Rogers JT, Moyers JP, et al. Prevalence and clinical course of pleural effusions at 30 days after coronary artery and cardiac surgery. Am J Respir Crit Care Med 2002;166:1567-71.

44. Labidi M, Baillot R, Dionne B, et al. Pleural effusions following cardiac surgery: prevalence, risk factors, and clinical features. Chest 2009;136:1604-11.

45. Heidecker J, Sahn SA. The spectrum of pleural effusions after coronary artery bypass grafting surgery. Clin Chest Med 2006;27:267-83.

46. Peng MC, Hou CJ, Li JY, et al. Prevalence of symptomatic large pleural effusions first diagnosed more than 30 days after coronary artery bypass graft surgery. Respirology 2007;12:122-6.

47. Stelzner TJ, King TE Jr, Antony VB, et al. The pleuropulmonary manifestations of the postcardiac injury syndrome. Chest 1983;84:383-7.

48. Engle MA, McCabe JC, Ebert PA, et al. The Postpericardiotomy syndrome and antiheart antibodies. Circulation 1974;49:401-6.

49. Imazio M, Brucato A, Rovere ME, et al. Colchicine prevents early postoperative pericardial and pleural effusions. Am Heart J 2011;162:527-32.e1.

50. Imazio $M$. The post-pericardiotomy syndrome. Curr Opin Pulm Med 2012;18:366-74.

51. McGrath EE, Blades Z, Anderson PB. Chylothorax: aetiology, diagnosis and therapeutic options. Respir Med 2010;104:1-8.

52. Riley LE, Ataya A. Clinical approach and review of causes of a chylothorax. Respir Med 2019;157:7-13.

53. Doerr CH, Allen MS, Nichols FC 3rd, et al. Etiology of chylothorax in 203 patients. Mayo Clin Proc 2005;80:867-70. 
54. Maldonado F, Hawkins FJ, Daniels CE, et al. Pleural fluid characteristics of chylothorax. Mayo Clin Proc 2009;84:129-33.

55. Agrawal V, Doelken P, Sahn SA. Pleural fluid analysis in chylous pleural effusion. Chest 2008;133:1436-41.

56. Nair SK, Petko M, Hayward MP. Aetiology and management of chylothorax in adults. Eur J Cardiothorac Surg 2007;32:362-9.

57. Huggins JT, Doelken P, Sahn SA, et al. Pleural effusions in a series of 181 outpatients with sarcoidosis. Chest 2006;129:1599-604.

58. Soskel NT, Sharma OP. Pleural involvement in sarcoidosis. Curr Opin Pulm Med 2000;6:455-68.

59. Nicholls AJ, Friend JA, Legge JS. Sarcoid pleural effusion: three cases and review of the literature. Thorax 1980;35:277-81.

doi: 10.21037/shc-21-11

Cite this article as: Li C, Kazzaz FI, Scoon JM, Estrada-YMartin RM, Cherian SV. Lymphocyte predominant exudative pleural effusions: a narrative review. Shanghai Chest 2022;6:5.
60. Valdés L, Huggins JT, Gude F, et al. Characteristics of patients with yellow nail syndrome and pleural effusion. Respirology 2014;19:985-92.

61. Maldonado F, Tazelaar HD, Wang CW, et al. Yellow nail syndrome: analysis of 41 consecutive patients. Chest 2008;134:375-81.

62. Nidus BD, Matalon R, Cantacuzino D, et al. Uremic pleuritis--a clinicopathological entity. N Engl J Med 1969;281:255-6.

63. Berger HW, Rammohan G, Neff MS, et al. Uremic pleural effusion. A study in 14 patients on chronic dialysis. Ann Intern Med 1975;82:362-4.

64. Gilbert L, Ribot S, Frankel H, et al. Fibrinous uremic pleuritis: a surgical entity. Chest 1975;67:53-6.

65. Rodelas R, Rakowski TA, Argy WP, et al. Fibrosing uremic pleuritis during hemodialysis. JAMA 1980;243:2424-5. 